\title{
Acquisition of spectrally resolved multimode far-fields from terahertz quantum cascade lasers
}

\author{
${ }^{1}$ Photonics Institute, TU Wien, 1040 Vienna, Austria \\ ${ }^{2}$ Center for Micro- and Nanostructures, TU Wien, 1040 Vienna, Austria \\ ${ }^{3}$ Austrian Academy of Sciences, 1010 Vienna, Austria \\ ${ }^{4}$ Institute of Solid State Electronics, TU Wien, 1040 Vienna, Austria
}

Sebastian Schönhuber1,2, Martin Brandstetter 1,2, Michael Krall1,2, Martin A. Kainz ${ }^{1,2}$, Hermann Detz $^{3}$, Tobias Zederbauer ${ }^{2,4}$, Aaron M. Andrews ${ }^{2,4}$, Gottfried Strasser ${ }^{2,4}$, and Karl Unterrainer ${ }^{1,2}$

Quantum cascade lasers (QCLs) are small and powerful sources covering a wide spectral range from infrared to terahertz $(\mathrm{THz})$ radiation. When fabricated in metal-metal ridge waveguides, the output aperture is typically very small compared to the long wavelength of $\mathrm{THz}$ radiation, therefore it is challenging to obtain collimated, directional emission from such devices.

Since numerous potential applications, ranging from imaging to spectroscopy, require a collimated emission, various concepts have been developed to optimize the far-field properties of THz QCLs.

For analysing the far field, most commonly used are either single-pixel detectors mounted on a translation stage or focal plane arrays. These measurements give information about the spectrally integrated far field only, however several optimization concepts require knowledge about individual spectral components of the far field. Obtaining this information using narrowband optical filters [1] or apertures [2] is possible, but time consuming and challenging.

The presented measurement technique [3] consists of a microbolometer focal plane array (FPA) placed inside the sample chamber of an FTIR spectrometer (see Fig. 1 (a)). The THz radiation emitted from the QCL is coupled into the FTIR via a parabolic mirror, guided through the spectrometer and recorded with either the internal DTGS detector or the FPA. Each pixel of the FPA records an individual interferogram, which is fourier transformed afterwards to obtain the spectrally resolved far field. In Fig. 1 (c) spectra obtained with the internal DTGS detector and the FPA are compared and show very good agreement.

Promising sources for multimode directional emission are THz quantum cascade random lasers [4] (Fig. 1 (b)). These devices allow the shaping of the far-field emission properties by changing the scatter pattern, with an optimized configuration collimated multimode emission is possible. With the presented technique, the contributions of the individual modes of $\mathrm{THz}$ random lasers to the integrated far field can be distinguished. In Fig. 1 (d), the far field contributions of two selected modes are depicted, in Fig. 1 (e) the integrated far field of the same device is shown.

(a)

(b)

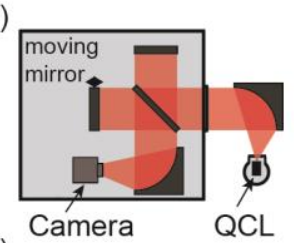

Surface emission

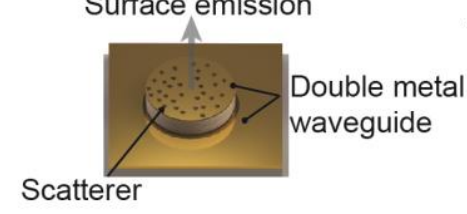

(c)

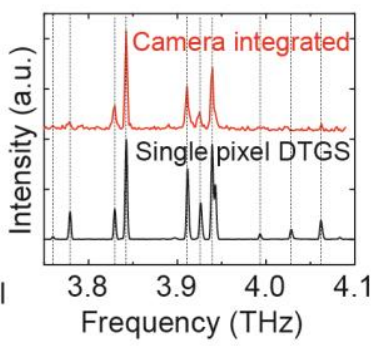

(d)

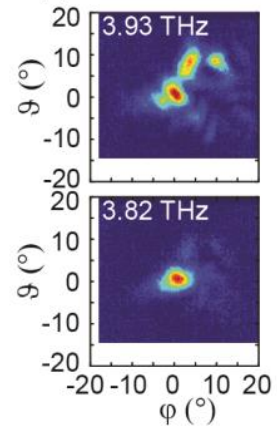

(e)

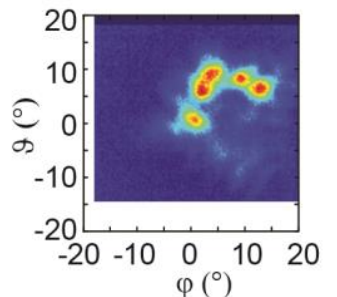

Fig. 1. (a) Scheme of measurement setup. The focal plane array microbolometer (camera) is placed in the sample chamber of an FTIR. (b) Scheme of THz quantum cascade random laser (c) Comparison of spectra obtained with the FTIR internal DTGS detector (bottom) and the microbolometer camera. Both exhibit the same spectral features. (d) Far-field contributions of two selected modes of a THz quantum cascade random laser. (e) Integrated far field of the device.

Using this technique, the properties of cavity concepts for broadband multimode emission, required for various applications of THz QCLs, can be studied accurately.

\section{References}

[1] J. He et al., "Narrow bandpass tunable terahertz filter based on photonic crystal cavity," Appl. Opt. 51, 776-779 (2012).

[2] A. A. Tavallaee et al., "Terahertz quantum-cascade laser with active leaky-wave antenna," Appl. Phys. Lett. 99, 141115 (2011).

[3] Brandstetter et al., "Spectrally resolved far-fields of terahertz quantum cascade lasers," Opt. Express 24, 25462-25470 (2016).

[4] Schönhuber et al., "Random lasers for broadband directional emission," Optica 3, 1035-1038 (2016). 
(C) ( 2017 IEEE. Personal use of this material is permitted. Permission from IEEE must be obtained for all other uses, in any current or future media, including reprinting/republishing this material for advertising or promotional purposes, creating new collective works, for resale or redistribution to servers or lists, or reuse of any copyrighted component of this work in other works.

This is the accepted version of S. Schönhuber et al., "Acquisition of spectrally resolved multimode farfields from terahertz quantum cascade lasers," 2017 Conference on Lasers and Electro-Optics Europe \& European Quantum Electronics Conference (CLEO/Europe-EQEC), Munich, 2017, doi :

10.1109/CLEOE-EQEC.2017.8086426 